\title{
Ultrasound in COVID-19
}

\author{
Goverdhan D Puri
}

Journal of Perioperative Echocardiography (2019): 10.5005/jp-journals-10034-1108

Bedside ultrasound has been integrated as a vital diagnostic and monitoring tool in most of the intensive care and emergency care units. The Society of Critical Care Medicine and American College of Emergency Physicians have provided guidelines for performing bedside ultrasound. ${ }^{1-3}$

Coronavirus disease-2019 (COVID-19) has been shown to have severe extrapulmonary manifestations owing to endothelial damage, dysregulation of the immune system, and maladaptation of ACE2-related pathways. ${ }^{4}$ The involvement of multiple systems in the disease process means that routine vitals monitoring and daily chest X-ray will seldom be enough to arrive at a definitive diagnosis when complications occur.

The case series' by Biswas et al. and Negi et al. highlight the role of bedside ultrasound in COVID-19. While the case series by Biswas et al. describes the utility of point-of-care ultrasound in general, Negi et al. describe the utility of echocardiography and guide on performing it. Bedside ultrasound has either helped guide the management or avoided further investigations in all these cases.

The first set of statements in the Society of Critical Care Medicine guidelines for ultrasound deals with extracardiac thoracic imaging which includes the diagnosis of pleural effusion, pneumothorax, and parenchymal lung diseases. Pleural effusion is an uncommon finding in COVID-19 unless there is associated superadded bacterial infection, hepatic, renal or cardiac dysfunction, or malignancy. ${ }^{5}$ Hence, the presence of pleural effusion in COVID-19 would imply a potentially treatable alternative diagnosis. On the other hand, pneumothorax is seen in around 1\% of COVID-19 admissions and is seen in spontaneously breathing patients as well. ${ }^{6}$ The timely diagnosis of this complication is important as it has been shown to not adversely affect the prognosis.

In abdominal imaging, the intensivist is trained to visualize the inferior vena cava, examine for free fluid, and image the bladder. Coronavirus disease 2019 can result in acute kidney injury in more than $50 \%$ of critical patients. ${ }^{7}$ Hyperpyrexia and gastrointestinal loss often result in hypovolemia which needs to be corrected expeditiously to avoid pre-renal damage. Volume resuscitation guided by dynamic indices of inferior vena cava dimension is crucial to achieve this. Apart from this, the presence of free fluid or abnormal appearance of the kidneys in COVID-19 patients could prompt evaluation by a radiologist.

Vascular ultrasound has an immense role to play in the management of COVID-19 patients. Around one in five critical COVID-19 patients have been identified to have thromboembolism and the mortality is also higher in these patients. ${ }^{8}$ Intensivists have $88 \%$ sensitivity and $98 \%$ specificity in identifying deep venous thrombosis when compared with ultrasound technicians. ${ }^{1}$ Right ventricle dilation and dysfunction are common in COVID-19 and have been found to occur with or without deep venous thrombosis.
Department of Anesthesia and Intensive Care, Postgraduate Institute of Medical Education and Research, Chandigarh, India

Corresponding Author: Goverdhan D Puri, Department of Anesthesia and Intensive Care, Postgraduate Institute of Medical Education and Research, Chandigarh, India, Phone: +91 9815199717, e-mail: gdpuri007@hotmail.com

How to cite this article: Puri GD. Ultrasound in COVID-19. J Perioper Echocardiogr 2019;7(2):27.

Source of support: Nil

Conflict of interest: None

Finally, ultrasound for procedural guidance is invaluable in the COVID-19 intensive care unit. The personal protective equipment often reduces the tactile perception and reduces the safety margin of landmark-based techniques.

Hence, the COVID-19 pandemic has highlighted the need for skill-based training in ultrasound for all personnel managing intensive care units.

\section{References}

1. Frankel HL, Kirkpatrick AW, Elbarbary M, et al. Guidelines for the appropriate use of bedside general and cardiac ultrasonography in the evaluation of critically III patients-part I: general ultrasonography. Crit Care Med 2015;43(11):2479-2502. DOI: 10.1097/ CCM.0000000000001216.

2. Levitov A, Frankel HL, Blaivas M, et al. Guidelines for the appropriate use of bedside general and cardiac ultrasonography in the evaluation of critically III patients-part II: cardiac ultrasonography. Crit Care Med 2016;44(6):1206-1227. DOI: 10.1097/CCM.0000000000001847.

3. American College of Emergency Physicians. American college of emergency physicians. ACEP emergency ultrasound guidelines-2001. Ann Emerg Med 2001;38(4):470-481. DOI: 10.1016/S01960644(01)70030-3.

4. Gupta A, Madhavan MV, SehgalK, etal. Extrapulmonarymanifestations of COVID-19. Nat Med 2020;26(7):1017-1032. DOI: 10.1038/s41591-0200968-3.

5. Garg M, Gupta P, Maralakunte M, et al. Diagnostic accuracy of CT and radiographic findings for novel coronavirus 2019 pneumonia: systematic review and meta-analysis. Clin Imaging 2020. 72:75-72:82.

6. Martinelli AW, Ingle T, Newman J, et al. COVID-19 and pneumothorax: a multicentre retrospective case series. Eur Respir J [Internet] 2020;56(5):Available from: http://dx.doi.org/10.1183/13993003.026972020.

7. Nadim MK, Forni LG, Mehta RL, et al. COVID-19-associated acute kidney injury: consensus report of the 25th acute disease quality initiative (ADQI) workgroup. Nat Rev Nephrol 2020;16(12):747-764. DOI: 10.1038/s41581-020-00356-5.

8. Malas MB, Naazie IN, Elsayed N, et al. Thromboembolism risk of COVID19 is high and associated with a higher risk of mortality: a systematic review and meta-analysis. E Clin Med 2020;29:100639. 